\title{
Quantitative sensory testing is feasible and is well-tolerated in patients with sickle cell disease following a vaso-occlusive episode
}

This article was published in the following Dove Press journal: Journal of Pain Research

\author{
Nitya Bakshi' \\ Ines Lukombo 1,2 \\ Inna Belfer ${ }^{3}$ \\ Lakshmanan Krishnamurti ${ }^{\prime}$ \\ 'Division of Pediatric Hematology- \\ Oncology, Department of Pediatrics, \\ Children's Hospital of Pittsburgh of \\ UPMC, Pittsburgh, PA, ${ }^{2}$ University \\ of Pittsburgh, ${ }^{3}$ Department of \\ Anesthesiology, University of \\ Pittsburgh, Pittsburgh, PA, USA
}

\begin{abstract}
Introduction: Sickle cell disease (SCD) is an inherited blood disorder characterized by abnormally shaped sickle cells. The hallmark of this disease is intermittent, painful vaso-occlusive episodes (VOE), but a subset of individuals with SCD experience chronic pain. The mechanism of transition to chronic pain is not well understood in SCD, but there is evidence of altered pain processing in individuals with SCD. The impact of VOE on pain sensitivity is not established. The objective of this study was to determine the feasibility and tolerability of quantitative sensory testing (QST) in SCD following a VOE to better understand the contribution of VOE to the development of chronic pain.
\end{abstract}

Methods: As part of a larger pain sensitivity study, pediatric patients with SCD were offered QST following a VOE-related Emergency Room visit or inpatient hospitalization. The feasibility of recruitment and completion of QST was measured, and tolerability of QST was determined using post-QST assessments of pain, and compared with measurements at steady state.

Results: Ten participants completed QST following a VOE. The median age was 16.5 , and $60 \%$ were female. Overall, 10 of 16 (62.5\%) patients approached for QST following VOE completed QST. This included 8 of 12 patients who had previously completed QST at steady state. There were no statistically significant differences in pain intensity and Gracely Box scores after QST following a VOE, when compared to steady-state QST.

Conclusion: QST is feasible and is well-tolerated following a VOE in patients with SCD. Large prospective studies are needed to determine the impact of VOE on experimental pain sensitivity and must take into account all factors contributing to pain sensitivity.

Keywords: quantitative sensory testing, sickle cell disease, pain

\section{Introduction}

Sickle cell disease (SCD) is an inherited blood disorder characterized by abnormally shaped sickle cells, associated with painful vaso-occlusive episodes (VOE) due to sickling. Intermittent, episodic pain is the hallmark of SCD, and usual sites of pain include the extremities, back, and chest. ${ }^{1}$ A subset of individuals with SCD experience frequent or daily pain. In the Pain in Sickle Cell Epidemiology Study (PISCES) - the largest natural history study of pain to date, individuals with SCD reported pain using diaries for 6 months, and $29.3 \%$ of adults experienced pain on $>95 \%$ of reported diary days, suggesting the presence of chronic pain. The mechanism of transition to chronic pain is not well described in SCD, but there is evidence of altered pain processing in individuals with SCD. Humanized mouse models of SCD recapitulate SCD pain, and mice have mechanical, thermal, and deep-tissue hyperalgesia, ${ }^{3}$ as well as hypersensitivity to
Correspondence: Lakshmanan Krishnamurti

Pediatric BMT, Aflac Cancer and Blood Disorders Center, Children's Healthcare of Atlanta, 2015 Uppergate Drive, Atlanta, GA 30322, USA

Email lkrishn@emory.edu 
cold stimuli. ${ }^{4}$ Children with SCD show increased sensitivity to cold pain ${ }^{5,6}$ and heat pain, ${ }^{6}$ but not to mechanical pain, ${ }^{6}$ as compared to race-matched controls of similar age. Similarly, adults with SCD have increased sensitivity to experimental pain, ${ }^{7}$ and manifest features of either central sensitization (CS) or peripheral sensitization (PS), or both. ${ }^{8,9}$

In addition to vaso-occlusion due to sickling of the RBCs, there is a complex cascade of events that occurs during VOE, ${ }^{10}$ driven, in part, by inflammation. As SCD is associated with inflammation, ${ }^{11}$ and pro-inflammatory cytokines are increased not only at steady state, ${ }^{12-14}$ but further increased during a VOE, ${ }^{15-17}$ it is plausible that sickling pain and inflammation play a role in CS or PS as well as the transition to chronic pain in SCD. In humanized mouse models of SCD, both mechanical and deep-tissue hyperalgesia increase with sickling. ${ }^{3}$ Thus, it is possible that sickling and/or inflammation during a VOE influence pain sensitivity in SCD. Experimental pain sensitivity in SCD has been studied at steady state, ${ }^{5,6}$ and in the presence of chronic pain in adults with SCD. ${ }^{9,18}$ Quantitative sensory testing (QST) during a VOE is untenable; however, testing following a VOE may provide insight into the role of VOE on pain sensitivity in SCD. To date, there have been no studies of experimental pain sensitivity following a VOE in children or adults. The feasibility, acceptability, and tolerability of such testing is, therefore, unknown. The objective of this study was to determine the feasibility and tolerability of QST in SCD following a VOE.

\section{Methods}

\section{Participant recruitment}

Participants of this study were patients at a comprehensive sickle cell clinic at a large children's hospital. This study was a sub-study of a previously published larger study of experimental pain sensitivity in patients with SCD and controls. ${ }^{19}$ Participants were eligible for, and offered participation in, the main study if they were between 8 and 21 years of age and had a diagnosis of SCD of any genotype, and did not have any exclusion criteria. Participants in the main study completed QST at steady state, that is, $>2$ weeks from their most recent hospitalization or Emergency Room (ER) visit for pain. Patients with SCD in the main study were excluded if they had 1) any other disease or sensory condition that could result in acute or chronic pain, 2) prior history of stroke, 3 ) any recent surgical procedures or pain interventional procedure in the preceding 3 months, 4) trauma or injury to the proposed testing sites, 5) significant cognitive impairment, or 6) active major psychiatric or mood disorder. For patients with SCD, who were receiving long-acting opioids or adjunctive medications for pain, suggesting they had features of chronic pain, steady-state testing was completed when they self-reported that they were experiencing baseline pain levels. Patients were offered participation in this sub-study if they were already enrolled in the main study or met criteria for the main study, and were within one week of discharge or were anticipating discharge following an ER visit or hospitalization for a VOE. Most of those offered participation were already enrolled in the main study and had completed steady-state QST; in a minority of instances, they had either been enrolled in the main study but not yet completed steady-state QST, or were simultaneously offered participation in the main study and this sub-study. Additional written informed consent from the patient or parent, as applicable, was obtained for this sub-study, and assent was obtained in the case of minors. Recruitment for the main study occurred between January 2013 and June 2014 and, for this sub-study, between September 2013 and June 2014.

The primary study objective of feasibility and tolerability of QST following VOE was determined using the following measures: 1) recruitment into this phase of the study, defined by the proportion of patients approached who were willing to consider this phase of the study; 2) completion of QST, defined by the proportion of patients who completed QST following a VOE; and 3) tolerability of QST following VOE as determined by pain intensity and Gracely Box scores following QST, with comparison to tolerability of QST at steady state. The study was determined feasible if at least $50 \%$ of those approached were able to complete testing within the study time window following a VOE. QST was determined to be tolerable if participants did not have post-QST pain intensity scores different from their steady-state testing values. Secondary objectives were to determine if there were any differences in experimental pain sensitivity following VOE when compared to the steady-state values.

The study was approved by the Institutional Review Board at the University of Pittsburgh, and written informed consent and assent was obtained prior to all procedures.

\section{Study procedures \\ Clinical characteristics}

SCD and pain-related clinical characteristics were abstracted from the medical record. The clinical burden of pain was measured by the number of visits for SCD pain to the ER, or inpatient admissions for pain over 6 months, 1 year, and 3 years prior to the steady-state QST assessments in the main study. Moreover, detailed data pertaining to the ER visit or hospitalization for VOE were abstracted from the medical record. 
Measures of psychological and pain-related functioning Prior to QST, participants completed patient-reported measures of psychological functioning and quality of life (QoL). These measures were identical to those completed at steady state. ${ }^{19}$ These were completed prior to QST to avoid any QSTrelated effects on these measures. Measures relevant to this study included: 1) Patient Reported Outcomes measurement Information System (PROMIS) measures of pain intensity, pain interference, anxiety, depressive symptoms, sleep, fatigue, and peer relationships to measure domains commonly affected by pain. Computerized Adaptive Testing (CAT) via the PROMIS Assessment Center ${ }^{20}$ was used for all domains except for pain intensity and sleep, for which short-form (SF) adult PROMIS were used because pediatric CAT versions are unavailable. All PROMIS questionnaires have a 7-day recall period and yield a T-score (a standardized score with a mean of 50 and standard deviation of 10) where higher T-scores indicate greater presence of a trait. The PROMIS pediatric item banks have undergone rigorous psychometric evaluation in children ages $8-17^{21-23}$ in the United States and are, furthermore, valid and responsive to pain in children with $\mathrm{SCD} .{ }^{24}$ 2) Catastrophizing, measured using the Pain Catastrophizing Scale (PCS), ${ }^{25,26}$ a 13-item questionnaire, which has been validated in children 8-16 years of age in community samples as well as in children with chronic pain. ${ }^{25-28}$ Total scores range from 0 to 52 , with higher scores indicating greater pain catastrophizing, and both total and subscale scores were calculated. 3) QoL, measured using the Peds $Q L \odot$ Generic $^{29-34}$ and SCD specific ${ }^{35,36}$ Quality of Life (QoL) modules, using a 1 -month recall period. Scores range from 0 to 100 , and higher scores represent better health-related (HR)-QoL.

\section{QST methods}

The QST methodology was identical to that done at steady state, and has been described in detail in our previous publication. ${ }^{19}$ Briefly, sensitivity to pressure, mechanical, and thermal pain was evaluated. The pain threshold was noted when the participant indicated this was when pain was first perceived, and pain tolerance was noted when pain was reported to be no longer tolerable. Pressure sensitivity was determined using a pressure algometer, mechanical sensitivity using pinprick probes, and thermal sensitivity (heat and cold) was determined using the TSA-II Neuroanalyzer. Mechanical and heat temporal summation (MTS and HTS, respectively) was determined using pinprick probes and TSA-II Neuroanalyzer, respectively; and pain scores were reported verbally on a $0-10$ scale. Parameters estimated were: pressure pain threshold (PPTh) and pressure pain tolerance (PPTo), mechanical pain threshold (MPTh), mechanical pain tolerance using pinprick probes (MPTo_probe) and using Von Frey Filaments (MPTo_VF), cold- and heat-detection thresholds (CDT and HDT), cold and heat pain thresholds (CPTh and HPTh) and heat pain tolerance (HPTo). MTS and HTS were estimated. Pain intensity scores, reported verbally by participants on a $0-10$ scale, were collected following completion of each sensory modality.

The immediate area involving the VOE site or around the VOE site was avoided, as well as the site(s) of intravenous lines, as both were areas where pain and discomfort could potentially be increased with QST. Participants were not asked to withhold or change pain medications prior to or during testing, including pain medications; however, details of medications were noted. As with the main study, participants were informed they could stop QST at any time. All procedures were demonstrated to participants prior to testing. QST was completed by a single investigator and data were not reviewed until all participants had been tested. Participants were seated comfortably in a chair for testing with both arms resting comfortably on an adjustable table. QST was conducted in a quiet area to minimize distractions. In addition, some participants consented to be in an ancillary study of facial expressions with laboratory pain, and their facial expressions were filmed during QST.

Timing of QST was driven by patient readiness and patient preference based on resolution of pain, and was done following discharge from the ER or the hospital, or a few hours prior to planned discharge, if requested as such by the participant. Patient readiness for QST and readiness for discharge from the hospital was used to determine timing of testing as most had previously undergone steady-state testing and were familiar with study procedures. Prior to discharge from hospital or following an ER visit for VOE, participants were offered participation in this study and, if they expressed interest, asked to indicate their preferred timing of testing based on self-assessment of pain resolution as well as patient preference and readiness for QST. As with QST at steady state, participants (and parent/guardian in case of minors) were informed of the potential risk for increased pain following QST. If they indicated interest in returning for QST following VOE, the study team contacted them and scheduled a QST session based on their preference. If the session could not be scheduled within a week of discharge, this was considered a non-completed QST, and not followed up further. If QST was conducted prior to discharge from the hospital, in addition to patient readiness and preference for timing of QST, the investigators based timing of testing on self-reported patient readiness for discharge and additionally reviewed the medical record for self-reported pain levels, trends in use of opioid 
analgesics and transition to $\mathrm{PO}$ analgesics, and ensured pain intensity scores on a scale of $0-10$ were not greater than $5 / 10$ prior to QST (unless a participant had chronic pain and where they reported that their non-VOE baseline pain intensity scores were $>5 / 10$ ). For patients with chronic pain, then, in addition to patient readiness for discharge and readiness for QST, it was only undertaken when the patient-indicated pain was at their baseline level. Additionally, for prospective participants who had had previously not undergone steady-state QST, QST was only done following discharge from the hospital - at a separate visit - and timing was based on patient readiness and pain resolution. QST was typically completed after-hours or on weekends to accommodate patient preference and convenience, at timings most convenient to patients, and often involved an additional, separate visit. All participants were provided compensation, in the amount of $\$ 25$ for participation in a QST session following a VOE, in addition to the $\$ 25$ that all participants received for completing QST at steady state.

\section{Post-QST assessments}

Following QST, participants completed the Situational Pain Catastrophizing Scale and the Gracely Box Scale for Pain Intensity and Unpleasantness. The Situational Pain Catastrophizing Scale is a six-item scale that measured catastrophizing in response to laboratory pain stimuli. ${ }^{37}$ The "Gracely Box Scales" measure pain intensity and pain unpleasantness; each is a 21-box vertical scale numbered 0-20, with numerical values for verbal descriptors of both pain intensity and unpleasantness, ${ }^{38}$ and higher scores representing greater unpleasantness and intensity. Participants were asked to think about the entire session while answering these questions to assess the intensity and unpleasantness of experimental pain stimuli during the QST session.

\section{Results}

\section{Recruitment and enrollment}

Thirty-one patients from the main study who completed steady-state QST were eligible for recruitment in this substudy if they presented to the ER or were hospitalized for pain. Fourteen of these 31 patients had at least one ED visit or hospitalization for SCD pain in the study period from September 2013 to June 2014. Of these 14 patients, two either could not be approached or were missed by the study team in the week following discharge from ED or hospital after the pain episode. Of the 12 patients approached, two patients refused testing following VOE, two verbally expressed interest in QST but were unable to come for testing during the study window, and eight completed QST following VOE.

Four patients were either 1) enrolled on the main study but had previously not undergone steady-state testing, or 2) were offered participation in the main study and sub-study, following a VOE episode. Of these four patients, two patients refused participation and two completed QST following VOE. Of the two who completed VOE testing prior to steady state, only one patient subsequently completed QST at steady state, and one patient was unable to be scheduled, on the basis of their convenience, for steady-state testing prior to June 2014. The recruitment and enrollment schema is depicted in Figure 1.

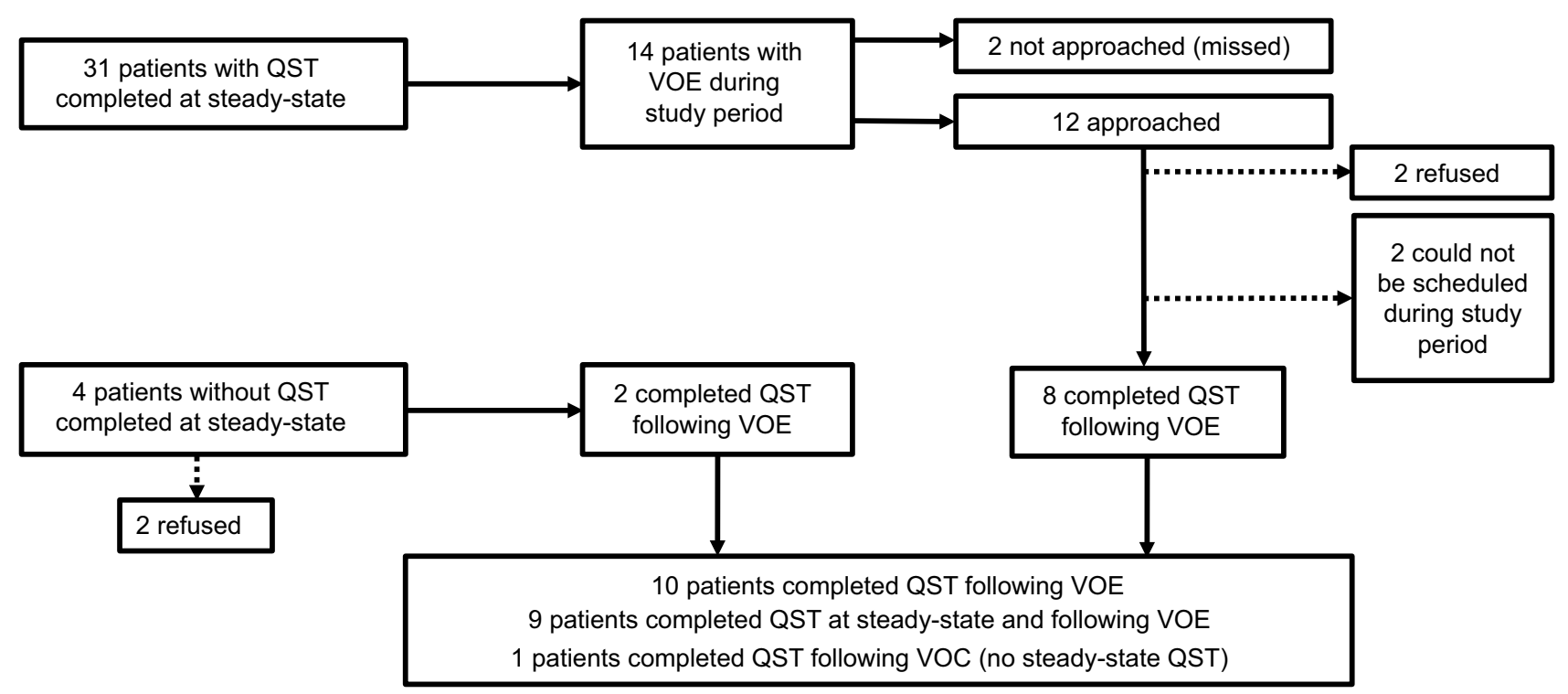

Figure I Recruitment and enrollment schema.

Abbreviations: QST, quantitative sensory testing; VOE, vaso-occlusive episode. 


\section{Timing of QST}

Based on their preference, QST was conducted after discharge in 6 of 10 participants, and prior to discharge in 4 participants. None of the participants who underwent testing prior to discharge had VOE involvement at proposed sites for QST. Of the participants who underwent QST prior to discharge from the hospital, two completed QST a few hours prior to discharge, on the day of discharge from the hospital. The other 2 participants also completed QST within a day of their planned discharge and when they indicated readiness for QST; however, their discharge occurred later than planned. Discharge was delayed in one of these participants for the treatment of a new lung infiltrate that met criteria for acute chest syndrome - a complication frequently associated with VOE, which requires treatment with antibiotics and red cell transfusion. The lung infiltrate was noted on chest X-ray prior to QST; however, its presence was not known to the research team prior to QST. In the second participant in whom discharge occurred later than planned, sites of pain included the abdomen and chest, where etiology of pain is potentially multifactorial. Delay in discharge in this patient was presumed by investigators to be, in part, due to ongoing pain management, although the clinical pain intensity score noted in the medical record in this participant following QST session was lower than pre-QST session pain intensity scores. One participant who completed QST following discharge, on the day of discharge from the ER, was re-admitted the following day. This patient had reported a pain intensity score of $0 / 10$ prior to discharge from the ER for pain, did not report pain prior to the QST session, and reported pain intensity of $0 / 20$ on the Gracely Box Scale following QST.

\section{Participant characteristics}

Demographic and psychological characteristics of all 10 participants are described in Table 1. The HR-QoL was worse following a VOE than at steady state. However, although catastrophizing scores were higher following VOE in the 9 participants who completed QST at steady state, this did not reach statistical significance. As expected, increased PROMIS pain intensity, pain interference, fatigue, and sleep disturbance scores were found when compared to steady state $(n=9)$.

\section{Clinical characteristics of participants}

Clinical characteristics of participants with SCD and the characteristics of pain management during ER visit or admission are described in Table 2 . The median duration of admission was nearly 4 days. Common sites of VOE included chest, back, lower extremities, and abdomen and, in 3 participants, also involved upper extremities. All participants received opioids and NSAIDs during their admission for VOE; two participants were also receiving gabapentin, and one of them was receiving methadone.

Table I Baseline demographic data and psychological characteristics

\begin{tabular}{|c|c|c|c|c|}
\hline & \multicolumn{2}{|l|}{ Following VOE } & \multicolumn{2}{|l|}{ Steady-state } \\
\hline & Median (IQR) & $\mathbf{n}$ & Median (IQR) & $\mathbf{n}$ \\
\hline Age & $16.5(11-20)$ & 10 & $16(10-19)$ & 9 \\
\hline Female sex, n (\%) & $6(60 \%)$ & 10 & $6(60 \%)$ & 9 \\
\hline $\begin{array}{l}\text { Quality of life: PedsQL }{ }^{\text {TM }} \text { Generic QoL inventory (total } \\
\text { score)** }\end{array}$ & $68(54.85-75)$ & 10 & $73.9(63.04-80.43$ & 9 \\
\hline $\begin{array}{l}\text { SCD-specific quality of life: PedsQL TM SCD module (total } \\
\text { score)** }\end{array}$ & $49.12(4 I .86-63.4 I)$ & 10 & $62.2(53.5-70.93)$ & 9 \\
\hline \multicolumn{5}{|l|}{ Pain Catastrophizing Scale } \\
\hline Total score & $30(24-3 I)$ & 10 & $24(19-29.5)$ & 8 \\
\hline Rumination & $12(10-13)$ & 10 & $12(10.5-15)$ & 8 \\
\hline Magnification & $6.5(3-7)$ & 10 & $4.5(3-6.5)$ & 8 \\
\hline Helplessness & $10.5(8-11)$ & 10 & $7(4.5-10)$ & 8 \\
\hline \multicolumn{5}{|l|}{ PROMIS measures (T-score) } \\
\hline Pain intensity* & $51.1(49.4-58.3)$ & 10 & $43.2(36.8-51.7)$ & 8 \\
\hline Pain interference $^{\wedge}$ & $59.1(52.7-63.2)$ & 10 & $51.4(49.5-56.05)$ & 8 \\
\hline Anxiety & $45.05(43.9-58.4)$ & 10 & $44.75(39.95-46.85)$ & 8 \\
\hline Depression & $51.5(46-56.2)$ & 10 & $47.35(45.05-51.5)$ & 8 \\
\hline Sleep disturbance ${ }^{\wedge}$ & $56.5(53.3-60)$ & 10 & 48.35 (46.4-5I.15) & 8 \\
\hline Fatigue $^{\wedge}$ & $59.3(55.3-63)$ & 10 & $49.25(41.75-54.1)$ & 8 \\
\hline
\end{tabular}

Notes: ${ }^{\wedge} 0.05<p<0.1 .{ }^{*} p<0.05$. ${ }^{*} p<0.01$ (Wilcoxon signed-rank test used participants with data at two time points).

Abbreviations: SCD, sickle cell disease; VOE, vaso-occlusive episodes; IQR, interquartile range; PROMIS, Patient Reported Outcomes Measurement Information System. 


\section{Feasibility of recruitment and completion of QST}

Overall, 10 of 16 participants approached for QST following VOE had completed QST, representing $62 \%$ of those approached. Among those who had previously completed QST at steady state, eight of 12 participants completed QST following VOE, representing $67 \%$ of those approached.

Table 2 Clinical and SCD pain characteristics

\begin{tabular}{|c|c|}
\hline Clinical characteristics & \\
\hline \multicolumn{2}{|l|}{ Genotype, n (\%) } \\
\hline $\mathrm{HbSS}$ & $6(60 \%)$ \\
\hline $\mathrm{HbSC}$ & $3(30 \%)$ \\
\hline HbS-beta+ thal & I (I0\%) \\
\hline Hydroxyurea use, n (\%) & $8(80 \%)$ \\
\hline \multicolumn{2}{|l|}{ Hematological parameters at admission, median } \\
\hline \multicolumn{2}{|l|}{ (IQR) } \\
\hline Hemoglobin $(g / d L)$ & $9.6(8.2-10.6)$ \\
\hline $\mathrm{MCV}(\mathrm{fL})$ & $92.9(84.6-99)$ \\
\hline WBC (thousand/mcL) & $11(8.1-14)$ \\
\hline Platelet count (thousand/mcL) & $339.5(292-514)$ \\
\hline Duration of hospital stay (days), median (IQR) & $3.9(3.7-5)$ \\
\hline Participants on methadone and/or gabapentin, $\mathrm{n}(\%)$ & $2(20 \%)$ \\
\hline \multicolumn{2}{|l|}{ Pain management during ER visit or hospital stay } \\
\hline Received opioids, n (\%) & $10(100 \%)$ \\
\hline Received NSAIDs, n (\%) & $10(100 \%)$ \\
\hline $\begin{array}{l}\text { Received naloxone (low dose for prevention of } \\
\text { pruritus), } \mathrm{n}(\%)\end{array}$ & $8(80 \%)$ \\
\hline \multicolumn{2}{|l|}{ SCD pain characteristics, median (IQR) } \\
\hline \multicolumn{2}{|l|}{ Number of pain episodes with ER or inpatient } \\
\hline \multicolumn{2}{|l|}{ admission prior to steady-state QST ( $n=9$ ) } \\
\hline 3 years prior to steady-state QST & $5(4-I I)$ \\
\hline I year prior to steady-state QST & $2(1-4)$ \\
\hline
\end{tabular}

Abbreviations: SCD, sickle cell disease; ER, Emergency Room; QST, quantitative sensory testing; MCV, mean corpuscular volume; WBC, white blood cell; NSAIDs, non-steroidal anti-inflamatory drugs.

\section{Tolerability of QST}

In general, QST following VOE was well tolerated, as measured by the Gracely Box scores, in all patients (Table 3). Moreover, Table 3 reports pain intensity scores, reported verbally by participants on a $0-10$ scale, following completion of each sensory modality. Further, pain intensity and Gracely Box scores during steady state are reported for comparison. The PPTh estimation on one side was omitted in two patients due to it being the site of VOE or IV cannula placement. Mechanical pain thresholds and tolerance using pinprick probes as well as MTS protocols were omitted in one participant per their request; this participant had vasoocclusive pain in the upper extremity. Thermal thresholds/ tolerance could not be completed in one patient as equipment was unavailable at that testing session. HTS was not completed in 5 patients, due to unavailability of equipment, temporary unavailability of software program required to administer HTS, or patient refusal.

\section{Experimental pain sensitivity in SCD following a VOE}

Experimental pain sensitivity results are reported in Table 4. Except for differences in the mechanical detection threshold, there were no statistically significant differences in experimental pain sensitivity following VOE and at steady state $(\mathrm{n}=9)$; however, the absolute magnitude of differences may not be of relevance, and the clinical significance of these differences is unknown.

Table 3 Tolerability of QST following VOE

\begin{tabular}{|c|c|c|c|c|}
\hline \multirow[t]{2}{*}{ Tolerability measures } & \multicolumn{2}{|l|}{ Following VOE } & \multicolumn{2}{|l|}{ Steady state } \\
\hline & (median, IQR) & $\mathbf{n}$ & (median, IQR) & $\mathbf{n}$ \\
\hline Pain score after estimation of PPTh (right) & $0(0-0)$ & 10 & $0(0-0)$ & 8 \\
\hline Pain score after estimation of PPTh (left) & $0(0-1)$ & 8 & $0(0-0)$ & 8 \\
\hline Pain score after estimation of PPTo (right) & $0.5(0-1)$ & 9 & $0(0-1)$ & 7 \\
\hline Pain score after estimation of PPTo (left) & $0.5(0-1)$ & 9 & $0(0-2)$ & 7 \\
\hline $\begin{array}{l}\text { Average pain score reported with probe weight at tolerance level } \\
\text { following estimation of MPTo_probe }\end{array}$ & $3.8(0-4)$ & 9 & $2.8(0-6.2)$ & 7 \\
\hline $\begin{array}{l}\text { Average pain score reported with VF filament weight at tolerance } \\
\text { level following estimation of MPTo_VF }\end{array}$ & $3.3(0.2-4)$ & 10 & $2.2(I-5)$ & 7 \\
\hline Pain score after estimation of CDT & $0(0-0)$ & 9 & $0(0-0)$ & 8 \\
\hline Pain score after estimation of CPTh & $0(0-1)$ & 9 & $0(0-1.5)$ & 8 \\
\hline Pain score after estimation of HDT & $0(0-0)$ & 9 & $0(0-0)$ & 8 \\
\hline Pain score after estimation of HPTh & $0(0-0)$ & 9 & $0(0-0.5)$ & 8 \\
\hline Pain score after estimation of HPTo & $0(0-0)$ & 9 & $0(0-0.5)$ & 8 \\
\hline Gracely Box score (unpleasantness) & $0.5(0-4)$ & 10 & $3.5(0.5-7)$ & 8 \\
\hline Gracely Box score (intensity) & $0(0-6)$ & 10 & $6.5(0-11)$ & 8 \\
\hline
\end{tabular}

Note: $p>0.1$ using Wilcoxon signed-rank test for available matched pairs.

Abbreviations: QST, quantitative sensory testing; VOE, vaso-occlusive episodes; PPTh, pressure pain threshold; PPTo, pressure pain tolerance; MPTo_probe, mechanical pain tolerance using pinprick probes; MPTo_VF, mechanical pain tolerance using Von Frey Filaments; CDT, cold-detection threshold; CPTh, cold pain threshold; HDT, heatdetection threshold; HPTh, heat pain threshold; HPTo, heat pain tolerance; IQR, interquartile range. 
Table 4 Experimental pain sensitivity following a VOE, and comparison with experimental pain sensitivity at steady state

\begin{tabular}{|c|c|c|c|c|}
\hline \multirow[t]{2}{*}{ QST measures } & \multicolumn{2}{|l|}{ Following VOE } & \multicolumn{2}{|l|}{ Steady state } \\
\hline & (median, IQR) & $\mathbf{n}$ & (median, IQR) & $\mathbf{n}$ \\
\hline \multicolumn{5}{|l|}{ Pressure (kgf) } \\
\hline Pain threshold (PPTh) & $1.253(0.88-2.34)$ & 10 & $1.263(0.76-1.433)$ & 9 \\
\hline Pain tolerance (PPTo) & $2.678(1.707-3.017)$ & 10 & $2.887(2.127-3.077)$ & 9 \\
\hline \multicolumn{5}{|l|}{ Mechanical } \\
\hline Detection threshold (MDT) $(\mathrm{g})^{*}$ & $0.111(0.069-0.196)$ & 10 & $0.067(0.05-0.096)$ & 9 \\
\hline Pain threshold (MPTh) $(\mathrm{mN})$ & $51.984(17.148-157.586)$ & 9 & $78.793(16-137.187)$ & 9 \\
\hline Pain tolerance using pinprick probes (MPTo_probe) $(\mathrm{mN})$ & $409.6(307.2-512)$ & 9 & $358.4(256-5 \mid 2)$ & 8 \\
\hline Pain tolerance using Von Frey filaments (MPTo_VF) (grams) & $174(84-300)$ & 10 & $211.2(51.1-300)$ & 8 \\
\hline \multicolumn{5}{|l|}{ Mechanical temporal summation (I 28-mN probe) } \\
\hline Average pain score prior to stimulus & $0(0-0)$ & 9 & $0(0-0)$ & 9 \\
\hline Average pain score after single stimulus & $0(0-1)$ & 9 & $0.6(0-1.8)$ & 9 \\
\hline Average pain score after train of 10 stimuli & $3.4(0-4.8)$ & 9 & $3.2(0.6-6.8)$ & 9 \\
\hline Average pain score 15 seconds after cessation of stimuli & $0(0-0.6)$ & 9 & $0(0-2)$ & 9 \\
\hline Average pain score 30 seconds after cessation of stimuli & $0(0-0)$ & 9 & $0(0-1)$ & 9 \\
\hline Non-painful sensations reported with stimuli, n (\%) & $2(22.2 \%)$ & 9 & $4 / 8$ present & 8 \\
\hline Mechanical temporal summation $(\Delta)$ & $2(0-3.4)$ & 9 & $2.2(0.8-4.4)$ & 9 \\
\hline Windup ratio & $3.3(1.66-7.4)$ & 4 & $3.2(3.1-4.9)$ & 5 \\
\hline \multicolumn{5}{|l|}{ Thermal $\left({ }^{\circ} \mathrm{C}\right)$} \\
\hline Cold detection threshold (CDT) & $30.75(30.45-30.775)$ & 9 & $30.8(29.65-31.075)$ & 9 \\
\hline Cold pain threshold (CPTh) & $20.5(11.775-23.05)$ & 9 & $22.6(16.525-25.25)$ & 9 \\
\hline Heat detection threshold (HDT) & $33.525(33.4-33.775)$ & 9 & $33.275(33.125-33.3)$ & 9 \\
\hline Heat pain threshold (HPTh) & $40.25(38.65-42.525)$ & 9 & $40.975(38.075-43.425)$ & 9 \\
\hline Heat pain tolerance (HPTo) & $44.6(41.025-46.3)$ & 9 & $42.075(39.7-44.85)$ & 9 \\
\hline \multicolumn{5}{|l|}{ Thermal temporal summation } \\
\hline Difference between average of I0th rating and average of Ist rating & $4.5(2.5-4.5)$ & 5 & $2.75(0-6)$ & 6 \\
\hline Difference between average of highest rating and average of Ist rating & $4.5(2.5-4.5)$ & 5 & $4.25(0-6.5)$ & 6 \\
\hline
\end{tabular}

Note: $*_{p}=0.01$, Wilcoxon signed-rank test for available matched pairs.

Abbreviations: IQR, interquartile range; QST, quantitative sensory testing; VOE, vaso-occlusive episodes.

\section{Discussion}

To our knowledge, this is the first study to report the feasibility and tolerability of QST in pediatric patients with SCD following a VOE. These results indicate that QST appears to be feasible and well-tolerated in patients who have experienced recent SCD-related vaso-occlusive pain. Several pediatric and adult studies of QST in SCD have indicated that QST is well tolerated. ${ }^{5,6}$ QST is well tolerated even in adults who face a significant burden of chronic pain. ${ }^{8,18}$ Studies in pediatric patients have been conducted at steady state. ${ }^{5,6}$ The finding that QST is tolerated following a VOE is critical in that it allows examination of pain sensitivity following a VOE and facilitates inquiry into mechanisms in SCD-related chronic pain.

Furthermore, these results bring to light logistical difficulties faced in attempting to conduct QST following VOE. Barriers for patients to return at a short interval following discharge following VOE include inconvenience of a repeat visit, transportation, and the burden imposed by a recent ER visit or admission to the hospital that may have already been disruptive to patients' lives. Patient readiness for discharge is increasingly employed as the marker of the end of VOE and is used as an endpoint in analgesic trials of $\mathrm{SCD},{ }^{39}$ as actual discharge from the hospital may be influenced by unrelated variables. At our institution, patients often were observed overnight after they indicated readiness for discharge. Patients who preferred to undergo QST before leaving the hospital requested that QST be undertaken the evening prior to or the day of the planned discharge. The limitation of this approach is that clinical complications may supervene and delay the planned discharge. Thus, despite the flexibility in scheduling QST according to patients' convenience, there are logistical patient-level barriers to completion of QST following a VOE. Whereas one patient was re-admitted for pain within a day of QST, it is unclear if QST procedures contributed to pain because the patient reported no pain prior to or following completion of QST; moreover, re-admission for pain after an ED visit or hospitalization for pain is well described in both adults and children with $\mathrm{SCD},{ }^{40,41}$ and are associated with multiple factors. ${ }^{41-44}$

While this study was not powered to detect differences in pain sensitivity following VOE, we are also cognizant of the limitations to using change in experimental pain sensitivity 
following a VOE as a model to study pain mechanisms in SCD because many factors impact the change in experimental pain sensitivity. The concomitant or recent use of painrelieving medications such as acetaminophen, NSAIDs, and opioids may alter pain sensitivity and may limit interpretation of findings on QST. ${ }^{45}$ The presence of opioid-induced hyperalgesia due to the recent administration of opioids may also limit interpretation of QST. This may be particularly relevant in patients on chronic opioids. ${ }^{18,46,47}$ Repeated measurements of pain sensitivity in an individual within a short timeframe have been found to be generally concordant with intra-class correlation values between measurements of up to $0.8 .{ }^{48}$ However, minor differences in pain sensitivity are perhaps not unexpected between any two QST measurements, and may be influenced by multiple patient factors that influence pain sensitivity. ${ }^{49}$ There is a lack of normative data on pain sensitivity, and the correlation between pain sensitivity and clinical pain is unclear in children with SCD. Thus, it is unclear, what the minimally significant difference in pain sensitivity is in patients with SCD. Furthermore, pain sensitivity in SCD is influenced by psychological factors such as anxiety, depression, catastrophizing, and somatization. ${ }^{19}$ As these psychological characteristics may worsen during a VOE, they could potentially influence pain sensitivity.

\section{Conclusion}

QST is feasible and is well-tolerated in patients with SCD, following a VOE. Large prospective studies are needed to determine the impact of VOE on experimental pain sensitivity and must take into account all factors contributing to pain sensitivity.

\section{Acknowledgments}

The authors thank patients and their families for their participation in this research study. The authors acknowledge Helen Shnol, BS, and Jodi Martin, BS, for their assistance as research coordinators during this study. Dr. Nitya Bakshi received funding from the Sickle Cell Disease Association of America Research Scholar Award. Dr. Nitya Bakshi also acknowledges the participation and contribution of the American Society of Hematology Clinical Research Training Institute Program 2013-2014. This study was funded in part by a pilot grant from the Vascular Medicine Institute (VMI) and Clinical and Translational Science Institute (CTSI) Pilot Grant Program at the University of Pittsburgh.

The authors thank the Mapi Research Trust for permission to use PedsQL ${ }^{\text {TM }}$ measures, C1998 JW Varni, Ph.D, all rights reserved, PedsQL ${ }^{\text {TM }}$ contact information and permission to use: Mapi Research Trust, Lyon, France, https://eprovide. mapi-trust.org/ and http://www.pedsql.org/index.html. The authors thank Dr. Michael Sullivan for permission to use the Pain Catastrophizing Scale in this study.

\section{Disclosure}

The authors report no conflicts of interest in this work.

\section{References}

1. McClish DK, Smith WR, Dahman BA, et al. Pain site frequency and location in sickle cell disease: the PiSCES project. Pain. 2009; 145(1-2):246-251.

2. Smith WR, Penberthy LT, Bovbjerg VE, et al. Daily assessment of pain in adults with sickle cell disease. Ann Intern Med. 2008;148(2):94-101.

3. Cain DM, Vang D, Simone DA, Hebbel RP, Gupta K. Mouse models for studying pain in sickle disease: effects of strain, age, and acuteness. Br J Haematol. 2012;156(4):535-544.

4. Zappia KJ, Garrison SR, Hillery CA, Stucky CL. Cold hypersensitivity increases with age in mice with sickle cell disease. Pain. 2014;155(12):2476-2485.

5. O'Leary JD, Crawford MW, Odame I, Shorten GD, McGrath PA. Thermal pain and sensory processing in children with sickle cell disease. Clin J Pain. 2014;30(3):244-250.

6. Brandow AM, Stucky CL, Hillery CA, Hoffmann RG, Panepinto JA. Patients with sickle cell disease have increased sensitivity to cold and heat. Am J Hematol. 2013;88(1):37-43.

7. Campbell CM, Carroll CP, Kiley K, et al. Quantitative sensory testing and pain-evoked cytokine reactivity: comparison of patients with sickle cell disease to healthy matched controls. Pain. 2016;157(4):949-956.

8. Ezenwa MO, Molokie RE, Wang ZJ, et al. Safety and utility of quantitative sensory testing among adults with sickle cell disease: indicators of neuropathic pain? Pain Pract. 2016;16(3):282-293.

9. Campbell CM, Moscou-Jackson G, Carroll CP, et al. An evaluation of central sensitization in patients with sickle cell disease. J Pain. 2016;17(5):617-627.

10. Manwani D, Frenette PS. Vaso-occlusion in sickle cell disease: pathophysiology and novel targeted therapies. Hematology Am Soc Hematol Educ Program. 2013;2013:362-369.

11. Hebbel RP, Osarogiagbon R, Kaul D. The endothelial biology of sickle cell disease: inflammation and a chronic vasculopathy. Microcirculation. 2004;11(2):129-151.

12. Qari MH, Dier U, Mousa SA. Biomarkers of inflammation, growth factor, and coagulation activation in patients with sickle cell disease. Clin Appl Thromb Hemost. 2012;18(2):195-200.

13. Garrido VT, Proença-Ferreira R, Dominical VM, et al. Elevated plasma levels and platelet-associated expression of the pro-thrombotic and pro-inflammatory protein, TNFSF14 (LIGHT), in sickle cell disease. Br J Haematol. 2012;158(6):788-797.

14. Lanaro C, Franco-Penteado CF, Albuqueque DM, Saad ST, Conran $\mathrm{N}$, Costa FF. Altered levels of cytokines and inflammatory mediators in plasma and leukocytes of sickle cell anemia patients and effects of hydroxyurea therapy. J Leukoc Biol. 2009;85(2):235-242.

15. Graido-Gonzalez E, Doherty JC, Bergreen EW, Organ G, Telfer M, McMillen MA. Plasma endothelin-1, cytokine, and prostaglandin E2 levels in sickle cell disease and acute vaso-occlusive sickle crisis. Blood. 1998;92(7):2551-2555.

16. Keikhaei B, Mohseni AR, Norouzirad R, et al. Altered levels of proinflammatory cytokines in sickle cell disease patients during vasoocclusive crises and the steady state condition. Eur Cytokine Netw. 2013;24(1):45-52.

17. Michaels LA, Ohene-Frempong K, Zhao H, Douglas SD. Serum levels of substance $\mathrm{P}$ are elevated in patients with sickle cell disease and increase further during vaso-occlusive crisis. Blood. 1998;92(9):3148-3151. 
18. Carroll CP, Lanzkron S, Haywood C, Jr, et al. Chronic opioid therapy and central sensitization in sickle cell disease. Am J Prev Med. 2016;51(1 Suppl 1):S69-S77.

19. Bakshi N, Lukombo I, Shnol H, Belfer I, Krishnamurti L. Psychological characteristics and pain frequency are associated with experimental pain sensitivity in pediatric patients with sickle cell disease. J Pain. 2017;18(10):1216-1228.

20. Gershon RC, Rothrock N, Hanrahan R, Bass M, Cella D. The use of PROMIS and assessment center to deliver patient-reported outcome measures in clinical research. J Appl Meas. 2010;11(3):304-314.

21. Irwin DE, Varni JW, Yeatts K, DeWalt DA. Cognitive interviewing methodology in the development of a pediatric item bank: a patient reported outcomes measurement information system (PROMIS) study. Health Qual Life Outcomes. 2009;7:3.

22. Irwin DE, Stucky B, Langer MM, et al. An item response analysis of the pediatric PROMIS anxiety and depressive symptoms scales. Qual Life Res. 2010;19(4):595-607.

23. Varni JW, Stucky BD, Thissen D, et al. PROMIS Pediatric Pain Interference Scale: an item response theory analysis of the pediatric pain item bank. J Pain. 2010;11(11):1109-1119.

24. Dampier C, Barry V, Gross HE, et al. Initial evaluation of the pediatric PROMIS(R) health domains in children and adolescents with sickle cell disease. Pediatr Blood Cancer. 2016;63(6):1031-1037.

25. Crombez G, Bijttebier P, Eccleston C, et al. The child version of the pain catastrophizing scale (PCS-C): a preliminary validation. Pain. 2003;104(3):639-646.

26. Sullivan MJL, Bishop SR, Pivik J. The Pain Catastrophizing Scale: development and validation. Psychol Assess. 1995;7(4):524-532.

27. Van Damme S, Crombez G, Bijttebier P, Goubert L, Van Houdenhove B. A confirmatory factor analysis of the Pain Catastrophizing Scale: invariant factor structure across clinical and non-clinical populations. Pain. 2002;96(3):319-324.

28. Parkerson HA, Noel M, Pagé MG, Fuss S, Katz J, Asmundson GJ. Factorial validity of the English-language version of the Pain Catastrophizing Scale - child version. J Pain. 2013;14(11):1383-1389.

29. Varni JW, Limbers CA. The PedsQL 4.0 Generic Core Scales Young Adult Version: feasibility, reliability and validity in a university student population. J Health Psychol. 2009;14(4):611-622.

30. Chen X, Origasa H, Ichida F, Kamibeppu K, Varni JW. Reliability and validity of the Pediatric Quality of Life Inventory (PedsQL) Short Form 15 Generic Core Scales in Japan. Qual Life Res. 2007;16(7): 1239-1249.

31. Chan KS, Mangione-Smith R, Burwinkle TM, Rosen M, Varni JW. The PedsQL: reliability and validity of the short-form generic core scales and asthma module. Med Care. 2005;43(3):256-265.

32. Varni JW, Seid M, Knight TS, Uzark K, Szer IS. The PedsQL 4.0 Generic Core Scales: sensitivity, responsiveness, and impact on clinical decision-making. J Behav Med. 2002;25(2):175-193.

33. Varni JW, Seid M, Rode CA. The PedsQL: measurement model for the pediatric quality of life inventory. Med Care. 1999;37(2): $126-139$.
34. Varni JW, Burwinkle TM, Seid M, Skarr D. The PedsQL 4.0 as a pediatric population health measure: feasibility, reliability, and validity. Ambul Pediatr. 2003;3(6):329-341.

35. Panepinto JA, Torres S, Varni JW. Development of the PedsQL sickle cell disease module items: qualitative methods. Qual Life Res. 2012;21(2):341-357.

36. Panepinto JA, Torres S, Bendo CB, et al. PedsQL sickle cell disease module: feasibility, reliability, and validity. Pediatr Blood Cancer. 2013;60(8):1338-1344.

37. Edwards RR, Smith MT, Stonerock G, Haythornthwaite JA. Pain-related catastrophizing in healthy women is associated with greater temporal summation of and reduced habituation to thermal pain. Clin J Pain. 2006;22(8):730-737.

38. Gracely RH, McGrath F, Dubner R. Ratio scales of sensory and affective verbal pain descriptors. Pain. 1978;5(1):5-18.

39. Telen MJ, Wun T, McCavit TL, et al. Randomized phase 2 study of GMI-1070 in SCD: reduction in time to resolution of vaso-occlusive events and decreased opioid use. Blood. 2015;125(17):2656-2664.

40. Ballas SK, Lusardi M. Hospital readmission for adult acute sickle cell painful episodes: frequency, etiology, and prognostic significance. $\mathrm{Am}$ J Hematol. 2005;79(1):17-25.

41. Sobota A, Graham DA, Neufeld EJ, Heeney MM. Thirty-day readmission rates following hospitalization for pediatric sickle cell crisis at freestanding children's hospitals: risk factors and hospital variation. Pediatr Blood Cancer. 2012;58(1):61-65.

42. Carroll CP, Haywood C Jr, Lanzkron SM. Examination of the patient and hospitalization characteristics of 30-day SCD readmissions. South Med J. 2016;109(9):583-587.

43. Brodsky MA, Rodeghier M, Sanger M, et al. Risk factors for 30-day readmission in adults with sickle cell disease. Am J Med. 2017;130(5): 601.e609-601.e615.

44. McMillan JE, Meier ER, Winer JC, et al. Clinical and geographic characterization of 30-day readmissions in pediatric sickle cell crisis patients. Hosp Pediatr. 2015;5(8):423-431.

45. Samuelsen PJ, Nielsen CS, Wilsgaard T, Stubhaug A, Svendsen K, Eggen AE. Pain sensitivity and analgesic use among 10,486 adults: the Tromsø study. BMC Pharmacol Toxicol. 2017;18(1):45.

46.Hooten WM, Mantilla CB, Sandroni P, Townsend CO. Associations between heat pain perception and opioid dose among patients with chronic pain undergoing opioid tapering. Pain Med. 2010;11(11):1587-1598.

47. Chen L, Malarick C, Seefeld L, Wang S, Houghton M, Mao J. Altered quantitative sensory testing outcome in subjects with opioid therapy. Pain. 2009;143(1-2):65-70.

48. Geber C, Klein T, Azad S, et al. Test-retest and interobserver reliability of quantitative sensory testing according to the protocol of the German Research Network on Neuropathic Pain (DFNS): a multi-centre study. Pain. 2011;152(3):548-556.

49. Gierthmühlen J, Enax-Krumova EK, Attal N, et al. Who is healthy? Aspects to consider when including healthy volunteers in QST-based studies - a consensus statement by the EUROPAIN and NEUROPAIN consortia. Pain. 2015;156(11):2203-2211.
Journal of Pain Research

\section{Publish your work in this journal}

The Journal of Pain Research is an international, peer reviewed, open access, online journal that welcomes laboratory and clinical findings in the fields of pain research and the prevention and management of pain. Original research, reviews, symposium reports, hypothesis formation and commentaries are all considered for publication
The manuscript management system is completely online and includes a very quick and fair peer-review system, which is all easy to use. Visit http://www.dovepress.com/testimonials.php to read real quotes from published authors. 